\title{
DESPRENDIMIENTOS ROCOSOS Y RUIDO TAFONÓMICO: TRABAJO EXPERIMENTAL EN EL ALERO 2 DE PUNTA PÓRFIDO, GOLFO SAN MATÍAS (PROVINCIA DE RÍO NEGRO, ARGENTINA)
}

\author{
ROCKFALL PROCESSES AND TAPHONOMIC NOISE: EXPERIMENTAL WORK \\ AT THE ALERO 2 IN PUNTA PORFIDO, SAN MATÍAS GULF \\ (RÍO NEGRO PROVINCE, ARGENTINA)
}

\author{
Eugenia Carranza $a^{1,2}$ y Marcelo Cardillo ${ }^{1,2,3}$
}

\begin{abstract}
La costa oeste norpatagónica presenta indicios de ocupaciones esporádicas al menos entre los 6000 y 900 años AP. En este trabajo se presentan los resultados de un primer acercamiento desde la tafonomía lítica al Alero 2 ubicado en la localidad arqueológica de Punta Pórfido. Las excavaciones realizadas sugieren que el alero posee una compleja historia formacional, dominada por procesos endógenos como la meteorización y la caída de rocas que, junto con la presencia de potenciales pseudoartefactos, plantean un relevante problema de investigación. El presente trabajo utiliza herramientas conceptuales y metodológicas derivadas de la tafonomía lítica y de la arqueología experimental para discutir el proceso de formación del registro lítico.
\end{abstract}

Palabras claves: experimentación, alero, pseudoartefactos, material lítico, Norpatagonia.

The North Patagonian west coast shows signs of sporadic occupations at least between 6000 and 900 years BP. In this paper, we present the results of a first approach from lithic taphonomy to Alero 2, located in the archaeological site of Punta Pórfido. The excavations carried out suggest that the rock shelter has a complex formational history, dominated by endogenous processes, such as weathering and rockfall processes that, together with the presence of potential pseudo-artifacts, pose a relevant research problem. The present work uses conceptual and methodological tools derived from lithic taphonomy and experimental archaeology to discuss the process of formation of the lithic record.

Key words: Experimentation, rock shelter, pseudoartifacts, lithic material, North Patagonia.

La localidad de Punta Pórfido se encuentra ubicada en el sector oeste del golfo San Matías, en la provincia de Río Negro, Argentina. Este sector de la costa ha sido interpretado como de uso poco intensivo por parte de los grupos humanos que la habitaron en el pasado (Borella y Cardillo 2011; Favier Dubois y Borella 2011; Favier Dubois et al. 2008). Sin embargo, en los últimos años el sector oeste de la costa ha sido explorado de forma intensiva con el fin de reevaluar esta hipótesis (Borella et al. 2015). Por ello y con el objetivo de comprender mejor la importancia de este sector de la costa durante el Holoceno Medio y Tardío, el trabajo se concentró en áreas que se destacan por una mayor densidad relativa de registro arqueológico, así como en la mayor variabilidad en las condiciones fisiogeográficas y en la disponibilidad de recursos para la subsistencia (Borella et al. 2015).
Una de estas áreas se corresponde con la Punta Pórfido, que se caracteriza por el predominio de afloramientos rocosos duros (rocas ígneas y metamórficas falladas y plegadas) de gran irregularidad y la baja presencia de mantos eólicos. El período geológico de formación se corresponde al Jurásico Superior (De Alba 1964). Estos afloramientos rocosos conforman aleros y oquedades que pudieron haber constituido reparos de los vientos costeros. También se constató la disponibilidad de agua de origen freático que se acumula en depresiones naturales del terreno, en sectores espacialmente acotados. Estas características podrían haber funcionado como atractores en el pasado. Una de las particularidades del Alero 2 de Punta Pórfido, es que gran parte del depósito parece estar conformado por el aporte endógeno de la roca de caja (por desprendimiento de clastos y bloques de distinto tamaño) la cual posee,

\footnotetext{
${ }^{1}$ Instituto Multidisciplinario de Historia y Ciencias Humanas (IMHICIHU), Buenos Aires, Argentina. carranza.e89@gmail.com; marcelo.cardillo@gmail.com

${ }^{2}$ CONICET Consejo Nacional de Investigaciones Científicas y Técnicas, Buenos Aires, Argentina.

${ }^{3}$ Facultad de Filosofía y Letras, Universidad de Buenos Aires, Buenos Aires, Argentina.
} 
en algunos sectores, propiedades aptas para la talla. Esta característica nos llevó a evaluar la presencia de "artefactos tafonómicos" o pseudoartefactos (sensu Borrazzo 2011), correspondientes a lascas grandes de volcanita ácida tanto en superficie como en los sondeos. Durante las excavaciones se observó que una parte del registro lítico recuperado no presentaba atributos tecnológicos claros. A esto se suma que, dichos materiales correspondían a la misma materia prima de baja calidad para la talla de la que está conformada el alero. Asimismo, artefactos de este tipo de materia prima se detectaron en muy bajas proporciones en muestreos realizados en contextos de superficie aledaños a la Punta Pórfido. Estas observaciones nos llevaron a identificarlos en una primera instancia como pseudoartefactos. Por ello, como parte de los estudios sistemáticos llevados a cabo en el área, se evaluaron los procesos de formación post depositacionales en el Alero 2 de Punta Pórfido, desde una perspectiva geoarqueológica y actualista, con el objetivo de identificar el ruido tafonómico (Borrero 2015) existente en el locus. Asimismo, nos interesa evaluar la equifinalidad ${ }^{1}$ (Lyman 2004) de los procesos de formación naturales y la conducta humana y distinguir artefactos culturales de aquellos producidos por agentes tafonómicos. Numerosas investigaciones sobre estudios actualísticos se ocuparon previamente de esta temática (Borrazzo 2011; Garvey y Mena 2016; Haynes 1973; Jackson y García 2005; Lin et al. 2017; Lubinski et al. 2014; Nash 1993; Prentiss et al. 2015; entre otros). Siguiendo los lineamientos planteados por estos autores, en este trabajo se busca identificar los efectos morfológicos de los procesos físicos, como así también la identificación de atributos y rasgos que nos permitan reconocer la variabilidad producto de los contextos y sus procesos.

Por consiguiente, los objetivos de este trabajo son lograr una mayor comprensión de los procesos de formación del registro lítico y de las estrategias de uso del borde costero rocoso en el golfo de San Matías en Río Negro, a través de la experimentación, el análisis tecnológico y el modelado estadístico (Árboles de Decisión y estadística descriptiva).

\section{Área de Estudio}

La costa rionegrina del Golfo San Matías se diferencia en dos sectores, norte y oeste, en base a características geológicas y geomorfológicas (Favier Dubois y Borella 2011). El sector oeste corre de norte a sur entre la localidad de San Antonio Oeste y Puerto Lobos, en el límite con Chubut (Figura 1). Su principal rasgo geológico es la presencia de la meseta de Somuncurá, una planicie estructural lávica que desciende hacia el mar en forma de pedimentos de flanco (González Díaz y Malagnino 1984). Esta costa ha sido propuesta como de uso ocasional por parte de los grupos humanos que habitaron este litoral (Favier Dubois y Borella 2011). Esta propuesta se basó en la homogeneidad ambiental general del área, que habría dado lugar a una variabilidad y disponibilidad menores de recursos, la escasez de reparos topográficos y la baja presencia de agua dulce.
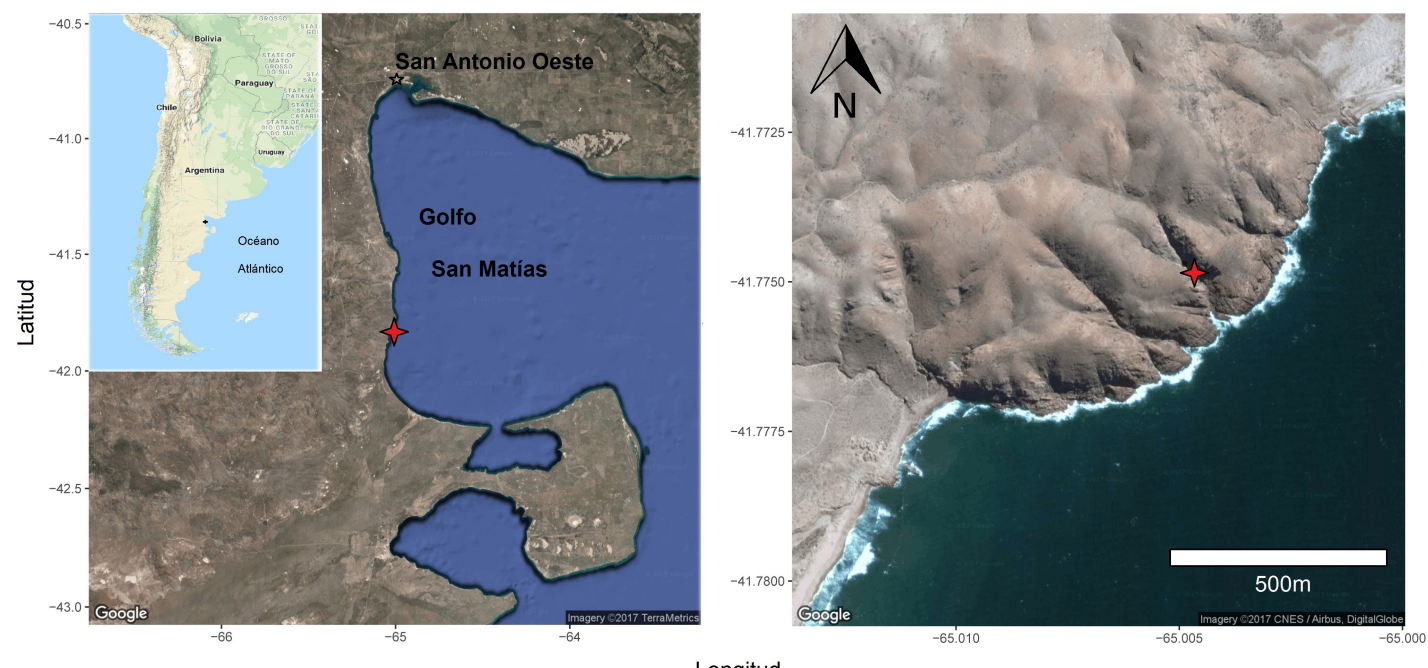

Longitud

Figura 1. Área de estudio (izquierda) y detalle del sector de Punta Pórfido (derecha).

Study area (left) and detail of the Punta Porfido sector (right). 
En la costa oeste se han localizado hasta el momento 42 loci, los cuales son más abundantes en el tramo septentrional, donde se registran dunas, cordones litorales y niveles aterrazados (Favier Dubois y Borella 2011; Favier Dubois et al. 2008). En general, el registro en esta zona es más visible debido a la escasez de los depósitos eólicos sobre las terrazas marinas (Favier Dubois et al. 2017; Manzi et al. 2009). En este trabajo nos centramos en el Alero 2 de la localidad arqueológica Punta Pórfido.

La localidad Punta Pórfido presenta un predominio de afloramientos rocosos duros (rocas ígneas y metamórficas falladas y plegadas) de gran irregularidad que forman pequeños aleros y oquedades distribuidos a lo largo de cañadones que llegan hasta la costa (Figura 2). Con el fin de buscar evidencias de ocupación humana en el pasado de estos sectores, se inició una exploración sistemática en búsqueda de materiales arqueológicos en superficie y también en estratigrafía, a través de pruebas de pala para identificar la existencia de depósitos en capa (Borella et al. 2015). En las prospecciones que se realizaron hasta unos $3,5 \mathrm{~km}$ al interior de los afloramientos rocosos, el Alero 2 es el único detectado hasta el presente que posee condiciones para contener depósitos arqueológicos, el resto de las estructuras reconocidas presentan tamaños muy pequeños y nula sedimentación.
El alero, que fue trabajado en dos oportunidades durante el 2017, constituye un espacio singular en la costa rionegrina y cuenta con un depósito sedimentario con potencial arqueológico. La altura del Alero 2 es de $10 \mathrm{~m}$ desde la línea de goteo, y se encuentra emplazado en un estrecho cañadón que desciende hacía el mar. Tiene un talud de $14 \mathrm{~m}$ de extensión, con una pendiente de $16^{\circ}$ y posee vegetación del tipo pastizales en su base, que funciona como trampa de material rocoso y sedimentario (Figura 2).

La materia prima que conforma el alero es riolita que presenta distintos grados de silicificación. En términos geoquímicos se trata de una volcanita ácida (posee un porcentaje mayor al $66 \%$ de óxido de silicio en su composición), y forma parte del grupo de las rocas ígneas volcánicas (volcanitas) (Alberti y Fernández 2015). En la porción sur de la costa oeste se identificaron afloramientos de rocas ígneas y metamórficas, donde se localizaron fuentes de materias primas efectivamente utilizadas y otras potenciales. Las que se encuentran en la localidad de Punta Pórfido corresponden a un asomo de la Formación El Jaguielito y Sector Cerrito (Alberti et al. 2015). Cabe destacar, además, que en la costa oeste presenta fuentes de materias primas tanto primarias como secundarias (Alberti et al. 2015).

En el área de estudio de este trabajo, la calcedonia y el sílex representan las principales materias primas

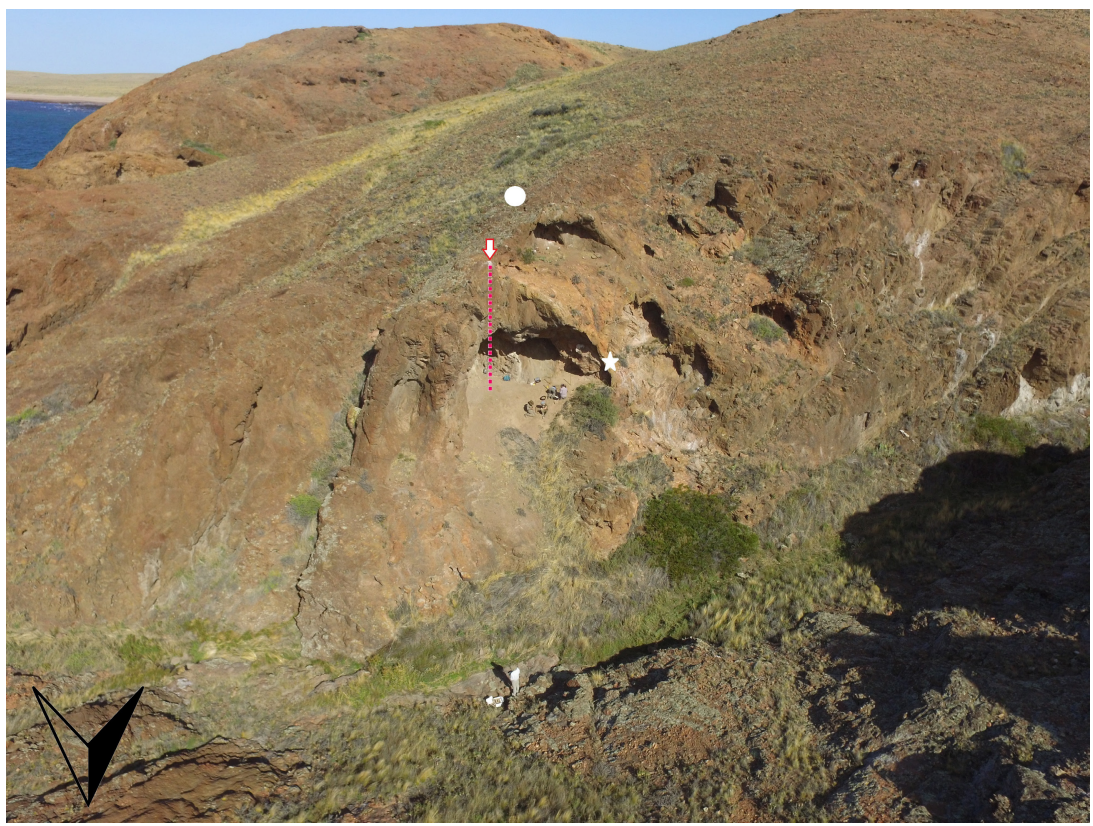

Figura 2. Detalle del alero y punto donde se realizaron las pruebas de caída (flecha) y la trayectoria y punto de impacto seleccionado. Los símbolos detallan los puntos de muestro de rocas empleadas para la caída y talla (círculo) y el afloramiento de mejor calidad empleado sólo en la talla (estrella).

Detail of the rock shelter and point where the fall tests were carried out (arrow) and the trajectory and point of impact selected. The symbols detail the sampling points of rocks used for the fall and knapping (circle) and the outcrop of better quality used only in knapping activity (star). 
explotadas para la confección de artefactos (Alberti et al. 2015). La tecnología de este sector costero está dominada por el subproducto de la talla de instrumentos, como ser lascas internas, externas y desechos no diferenciados. En menor medida se han recuperado instrumentos de formatización sumaria (predominan los filos simples) y los filos naturales con rastros complementarios. En cuanto a los núcleos, se registraron principalmente de tipo poliédrico, seguidos por los de lascados aislados (Cardillo y Scartascini 2011).

En el año 2017 se llevaron a cabo dos sondeos, de 1 $\mathrm{m} \times 60 \mathrm{~cm}$, alcanzando el metro y medio de profundidad mediante niveles artificiales de $10 \mathrm{~cm}$, en ambos casos se detectaron dos capas portadoras de lentes carbonosas a los 50-60 y 90-100 cm de profundidad, respectivamente. De éstas se obtuvo un fechado de $4560 \pm 100$ sobre valvas de Aulacomya ater en el caso de la primera, y otro de $6624 \pm 31$ en la segunda sobre carbón (Tabla 1), la calibración se realizó con el programa Calib 7.1 y las curvas SHCal13; (Hogg et al. 2013) para el hemisferio sur en el caso de los carbones y Marina 13 con efecto reservorio en el caso de las valvas, siguiendo lo sugerido por Favier Dubois y Jull (2018). En ambas excavaciones se recuperaron restos de carbón, escasos restos faunísticos y líticos los cuales se encontraban contenidos en las capas mencionadas, resultando estériles los niveles excavados entre ellas. A poco más de un metro de profundidad ambos sondeos alcanzaron la roca base del alero.

Tabla 1. Fechados radiocarbónicos.

Radiocarbon dates.

\begin{tabular}{lcc}
\hline \multicolumn{1}{c}{ Código } & Edad C14 AP & Edad cal. AP 2 $\sigma$ \\
\hline LP 3529 & $3680 \pm 90$ & $2105-1551$ \\
D-AMS 026733 & $6624 \pm 31$ & $5615-5584$ \\
\hline
\end{tabular}

\section{Aleros, Procesos de Formación y Pseudoartefactos}

Los aleros, siguiendo a Waters (1992), son rebajes formados naturalmente, superficiales bajo una roca sobresaliente. Sus bocas están expuestas al exterior, esto los hace una trampa efectiva de sedimentos tanto exógenos como de la propia meteorización que desintegra la roca que los conforma.

Las cuevas y aleros son ambientes interesantes para estudiar regional y localmente los procesos de formación, ya que proveen información no solo de la profundidad temporal representada en su tasa de depositación, sino también de los agentes tafonómicos que actúan modificando su estructura y por tanto el potencial arqueológico que contienen.
En este sentido, estimar la tasa de depositación predominante mediante la representación relativa de materiales autóctonos, provenientes del interior del alero (techo y paredes) y alóctonos, provenientes del exterior (p.ej., viento, agua), indican las probabilidades de preservación del registro arqueológico (Favier Dubois 1997).

Generalmente, los aleros poseen una plataforma de talud que se extiende hacia abajo desde la entrada. $\mathrm{El}$ agente principal que actúa sobre la pendiente es la gravedad, provocando el movimiento gradual de partículas sobre la pendiente del talud (Waters 1992; Wood y Johnson 1982). Este material suele estar poco seleccionado y está dominado por una serie de factores como el ángulo de pendiente; el tipo, magnitud y frecuencia de los procesos que causan el arrastre; la presencia y tipo de vegetación; tamaños de las partículas movilizadas; y contenido de humedad (Waters 1992).

También los procesos de alteración como la meteorización química y física de la roca que cae e impacta en el sustrato conforman depósitos de cascajo que pueden presentar algunos atributos que resultan similares a los producidos por la percusión humana. Lo que lleva a evaluar la posibilidad de que los humanos hayan utilizado este material de modo inmediato con o sin modificaciones (Nash 1993). Sin embargo, este autor advierte que las cuevas y aleros no representan un mayor problema en la identificación de artefactos tafonómicos y antrópicos, ya que estas geoformas están generalmente conformadas por rocas sedimentarias o de baja calidad para la talla (Nash 1993). En el caso del Alero 2, la volcanita ácida posee grados distintos de silicificación, esto nos llevó a preguntarnos cuál era el potencial de esta roca para generar pseudoartefactos a partir de su desprendimiento natural. Creemos que es posible que la fragmentación de las rocas por meteorización de la estructura interna del alero conforme depósitos de litos que presenten atributos comparables a los artefactos líticos producidos por humanos (materiales líticos de origen tafonómico o pseudoartefactos, sensu Borrazzo 2011). Consecuentemente, la caracterización y análisis tecno-morfológico y tafonómico del material recuperado nos llevó a preguntarnos sobre el origen de algunos desechos hallados en el contexto del sondeo.

La búsqueda de atributos que den cuenta de su origen, junto con el estudio de la organización tecnológica a nivel regional, es de los aspectos fundamentales para alcanzar una interpretación lo más ajustada posible al contexto. Por este motivo se desarrolló un protocolo experimental replicando la caída (desprendimiento natural) y la talla controlada de nódulos, a fin de evaluar las cualidades de la materia prima, conocer sus atributos y su potencialidad, tal como se explica a continuación. 


\section{Materiales y Métodos}

La muestra analizada proviene tanto de materiales arqueológicos como experimentales. En ambos casos se siguieron los mismos principios del análisis tecno-tipológico (Aschero 1983), lo que incluye a aquéllos recuperados durante la fase de excavación que fueron identificados como potenciales pseudoartefactos (sensu Borrazzo 2011).

\section{El experimento}

En este trabajo, buscamos evaluar el mecanismo y la dinámica de fractura de la roca de caja y el aporte endógeno del alero a la formación de material lítico natural. Es decir, evaluar el ruido tafonómico del locus y distinguir entre artefactos culturales de aquellos producidos por agentes naturales a través de un experimento para replicar in situ el desprendimiento de rocas y su impacto sobre el sustrato.

Para llevar adelante el experimento, se recolectaron 30 rocas provenientes de la parte superior del alero, consistentes en nódulos de obtención inmediata y que no han sufrido caída producto del desprendimiento (Figura 2). Para operativizar y controlar el impacto de cada nódulo, se crearon tres categorías de agrupamiento por tamaño y peso (chicos, medianos y grandes). Los rangos son: chico desde 100 g.; mediano $200 \mathrm{~g}$ a $500 \mathrm{~g}$.; grande $500 \mathrm{~g}$ a $1 \mathrm{~kg}$. Cada pieza fue cubierta en su totalidad con pintura de color con el objetivo de identificar los rasgos que puedan provocarse en la caída, así como también aumentar la obtrusividad del material experimental en el contexto de sitio. Cabe aclarar que la capa de pigmento de base acrílica utilizada no agrega tenacidad a la superficie de la roca.

Se registró la materia prima de los nódulos experimentales, largo ( $\mathrm{mm})$, ancho ( $\mathrm{mm})$, espesor $(\mathrm{mm})$ y peso $(\mathrm{g})$, altura del alero, la superficie/sustrato de impacto, presencia de vegetación y ángulo de la pendiente de impacto.

Para la experimentación propiamente dicha la altura de caída fue fijada en $10 \mathrm{~m}$, dentro del talud del alero a $1,20 \mathrm{~m}$ de la pared actual, sobre el techo del alero. Se tuvo en cuenta que, debido a la evolución del alero y el proceso de sedimentación a lo largo del tiempo, estas condiciones pueden haber sido distintas al registrado actualmente. Sin embargo, si se tiene en cuenta que entre las capas que provienen estos materiales hay una diferencia de $60 \mathrm{~cm}$, la altura máxima no habría variado considerablemente. Cada guijarro fue soltado en caída libre, sin ningún tipo de impulso sobre aproximadamente un mismo punto predeterminado, además algunas de ellas fueron registradas en video para poder reconstruir con detalle las trayectorias (Figura 3). Posteriormente, se realizó un registro del impacto, el desplazamiento de las piezas en el talud, fragmentación y rasgos macroscópicos resultantes. Se midió la distancia de desplazamiento (si la hubiese) entre el punto de impacto y el de depositación final. En caso de observar fracturas, se recolectaron todos los fragmentos generados. El material fue luego embalado por separado, para el análisis en el laboratorio. Para el análisis de las piezas experimentales se definieron las siguientes variables dicotómicas:

Presencia o ausencia de esquirlas. Se entiende estas como astilla o fragmento, generalmente alargados y puntiagudos, que se desprenden de la roca.

Presencia o ausencia de melladuras. Pequeñas hendiduras o grietas que se forman en el borde, particularmente en el filo de un instrumento, al impactar o romperse.

Fracturas de las piezas. Con el fin de comprender mejor las propiedades mecánicas y el proceso deformación de lascas con esta roca, se realizó una talla controlada sobre una submuestra del material experimental. Se tallaron tres bloques, dos en el laboratorio -trabajados en material de la sección superior del alero- y uno en el campo, éste último a partir de material del alero que forma parte de una veta de volcanita ácida de mayor calidad que aflora en la pared este del mismo. En todos los casos se utilizó percusión directa con el fin de extraer lascas utilizando las plataformas naturalmente disponibles en cada nódulo. La talla continuó hasta la pérdida de plataformas aptas para la extracción de lascas o cuando las fracturas en el nódulo hicieron inviable continuar la reducción. En el caso del nódulo de mayor calidad, uno de los fragmentos de mayor tamaño residuales fue empleado para la técnica de talla bipolar. En todos los casos se emplearon percutores duros de entre 9 y $10 \mathrm{~cm}$.

Todos los desechos generados fueron recuperados y analizados en el laboratorio, donde se separaron aquellos con atributos de lascas o vinculados al proceso de talla, como la identificación de plataformas de lascado. Esto permitió cuantificar la cantidad de lascas obtenidas en relación con la totalidad de desechos, así como el registro cualitativo de otros aspectos vinculados a la reducción lítica (comportamiento del material).

\section{Las muestras}

\section{Arqueológica}

En la muestra arqueológica, compuesta por $\mathrm{n}=25$ casos, se determinó también la materia prima y se registraron las variables tafonómicas observables. Se recuperó material lítico en superficie y en uno de los sondeos antes mencionados. Dado lo acotado de esta muestra, en este trabajo todos los materiales son analizados conjuntamente, sin discriminar su procedencia. 


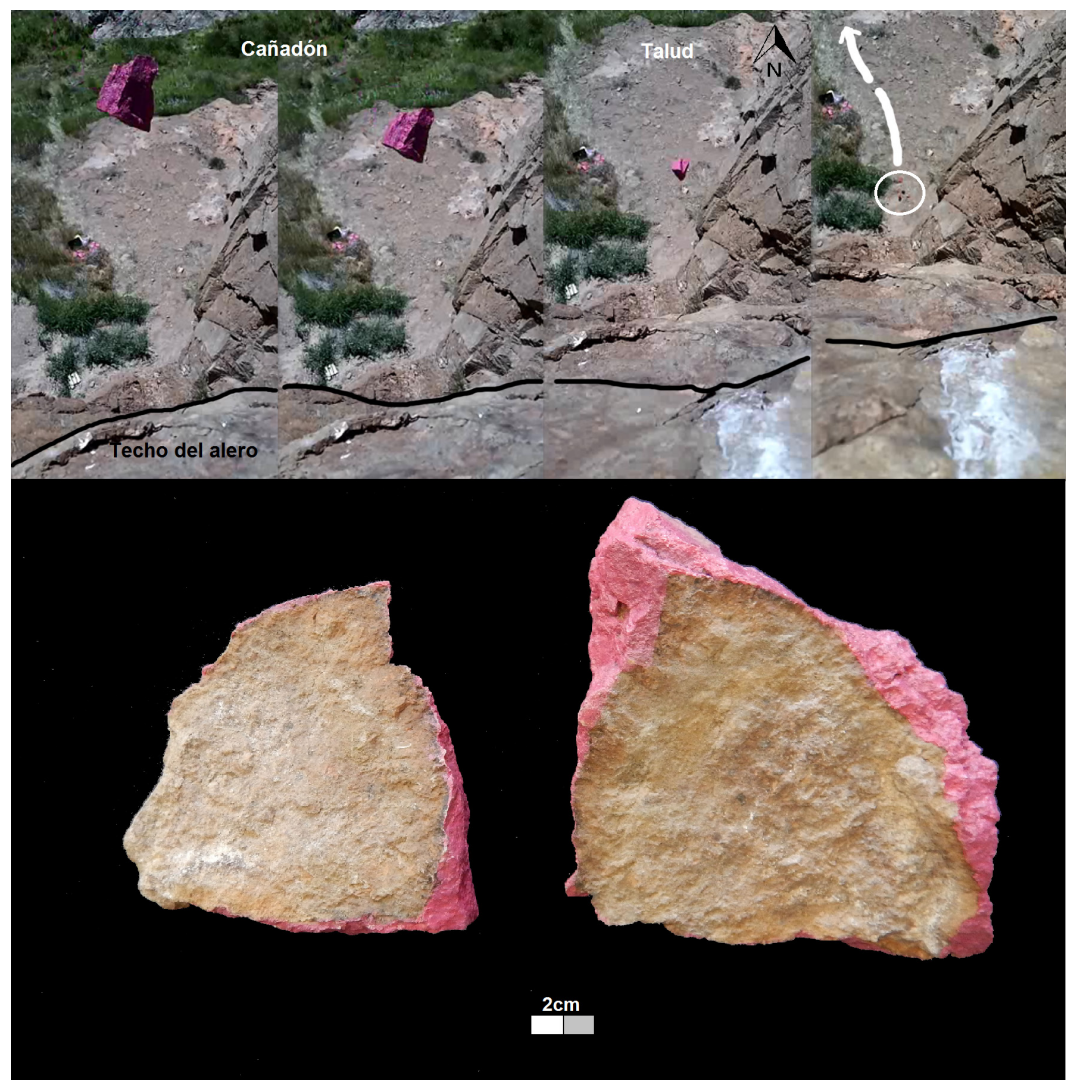

Figura 3. Registro de la caída desde el techo del alero (arriba) y nódulo fracturado por impacto (abajo).

Record of the fall from the roof of the rockshelter (top) and nodule fractured by impact (bottom).

\section{Experimental}

La muestra experimental está compuesta en total por 30 bloques de roca, pertenecientes a tres categorías de tamaño previamente definidas, perteneciendo 10 a cada una de ellas. Estos bloques fueron recolectados sobre el afloramiento rocoso asociado al techo del alero, se buscó que éstos presentaran la máxima similitud macroscópica en su composición y granulometría con el material recuperado dentro de éste, ya que como se ha observado (ver Infra) esta presenta variaciones, incluso dentro del mismo alero.

\section{Análisis estadísticos}

\section{Árboles de decisión}

Con fin de modelar la relación entre los agentes tafonómicos, las variables métricas de los nódulos experimentales y las trazas generadas por el proceso de caída, se aplicaron árboles de decisión, utilizando el método de Fast and Frugal Decision Trees (FFT). En general los árboles de decisión se basan en algoritmos diseñados para establecer parámetros clasificatorios a partir de una variable dependiente binaria (presenciaausencia, verdadero-falso, como el CART, Random Forest, C50, FFT, entre otros). En este aspecto, el trabajo pionero de Weitzel et al. (2014) aplicando árboles de decisión para predecir umbrales de fractura de artefactos por pisoteo en distintos contextos, muestra que éstos generan expectativas robustas para la probabilidad de daño en las lascas. Si bien los distintos métodos pueden variar en los algoritmos empleados, el objetivo es el mismo: particionar la variable dependiente a partir de un conjunto de variables independientes determinando umbrales clasificatorios y representándolo en forma de árbol. La estructura del árbol de decisión puede describirse a partir de sus nodos y sus ramas (Song y Ying 2015). Los nodos contienen la respuesta binaria (por ejemplo, con rastros-sin rastros, entero-fracturado). Su origen es el nodo central o raíz que representa a la variable explicativa de mayor importancia, seguido por otras de menor valor explicativo. Sólo una decisión es posible en la raíz y en los nodos intermedios, mientras que en el último nodo ambas decisiones se expresan conjuntamente (Song y Ying 2015). Sin embargo, 
uno de los aspectos más relevantes de los árboles de decisión no es solo la descripción de los patrones observados, sino la predicción. Los FFT, a diferencia de otros métodos similares no se sobreajustan a los datos, generando modelos explicativos algo más generales (Gigerenzer y Todd 1999; Phillips et al. 2017), lo que los convierte en buenas herramientas predictivas (y no sólo descriptivas). Otra particularidad es que se basan en un algoritmo no compensatorio, que a diferencia de otros métodos como la regresión logística (también empleada en el contexto de toma de decisiones), no toma en cuenta todas las variables independientes, también denominadas "señales" (cues) en este contexto. Una vez que una variable es seleccionada a un nivel de decisión determinado, no vuelve a ser utilizada, esto vuelve a este árbol de decisión "frugal", en términos de las variables que precisa para generar un modelo explicativo. Las ventajas relativas de este método en este tipo de contextos, ha sido demostrada por Phillips et al. (2017), entre otros.

Con la aplicación de los árboles de decisión esperamos generar el modelo más parsimonioso para explicar los patrones de daño generados experimentalmente. En estos métodos, es posible particionar la muestra en dos subpoblaciones y utilizar el árbol en una fase de aprendizaje y otra fase de contraste para evaluar independientemente su eficacia. En este caso, dado que la muestra experimental es de un tamaño moderado, se empleó en su totalidad para generar la predicción. Adicionalmente, se utilizaron distintos índices para evaluar la efectividad del modelo, los cuales se calculan a partir de una matriz de confusión. Cada fila de la matriz representa el conteo de los resultados predichos $v s$. los valores observados para la variable dependiente. Dada que la respuesta es dicotómica, la predicción correcta es denominada sensibilidad (tasa de aciertos), mientras que el rechazo correcto se denomina especificidad (tasa de rechazo). El modelo más eficiente será aquel que pueda predecir cada uno de los estados de la variable dependiente con el menor error posible.

Además de la sensibilidad y la especificidad, se estimó la precisión media del modelo, que promedia sensibilidad y especificidad, cuanto mejor sea el ajuste global, los valores estarán cercanos al 100\%. Por consiguiente, la sensibilidad (sens) representa el porcentaje de casos con criterio positivo (en este caso presencia de rasgos) que son evaluados correctamente por el modelo y se calcula: sens=hi/ (hi+mi; hi, (hi tasa de aciertos, mi tasa de fallas). La especificidad (esp) o tasa correcta de rechazo (identificación de casos sin rastros) es: esp=cr/fa+cr, (cr número correcto de rechazos, fa, falsas alarmas o rechazos erróneos). Por último, la precisión promedio (wacc) considera la relación entre ambos índices, en este caso se tomó la precisión promedio ponderada (wacc o weighted accuracy) estimada como: sens $\times \mathrm{w}+\mathrm{esp} \times \mathrm{w}$ (donde w es el peso por el que se pondera cada medida de ajuste $y$ varía entre 0 y 1 , por defecto; la ponderación es la misma para cada estimación 0,5 )

\section{Análisis y Resultados}

\section{Caída}

Durante la experimentación se registraron principalmente desplazamientos por saltación y rodamiento. Se observó un comportamiento de desplazamiento variable de los nódulos al impactar sobre la superficie del talud desde 0 hasta un máximo de 13,5 $\mathrm{m}$ y un promedio de 4,52 m, sólo un 3\% de los nódulos no mostró ningún desplazamiento. La correlación ( $\mathrm{r}$ de Pearson) entre las variables métricas de los nódulos y la distancia de desplazamiento sugiere que esta está vinculada positivamente al espesor $(\mathrm{r}=0,47, p=0,01)$.

La máxima distancia de desplazamiento alcanzado fue el cañadón en la base del talud cubierto de vegetación. Parte de los nódulos (4\%) fueron total o parcialmente entrampados por ésta (Figura 3). Como resultado del impacto un $94 \%$ de las piezas presentaron indicios de daño sobre su superficie, siendo el más común las melladuras $(n=28,94 \%)$ seguido por las esquirlas $(n=18$, $60 \%$ ) y las fracturas $(n=3,10 \%)$ (Figura 3 ). Sin embargo, este último rasgo estaba también presente en los nódulos previamente a la caída, por lo que no se vincula directamente con el impacto y rodamiento (Figura 3). Asimismo, todas las rocas que presentan esquirlamiento también sufrieron melladuras, mientras que un $n=10$ $(35 \%)$ presenta melladuras sin esquirlamiento.

Se recuperaron seis esquirlas producto del impacto de los nódulos experimentales. El tamaño máximo registrado es de $1,5 \mathrm{~cm}$, estas no presentan atributos ni rasgos semejantes a las extracciones producto de la talla controlada. Sugerimos que este material producto del desprendimiento podría estar aportando a la tasa de sedimentación del sitio y del talud; ver más adelante.

En cuanto al material fracturado por impacto $(n=3$, $10 \%$, Figura 3), es posible que estas fracturas ocurrieran a partir de las fisuras preexistentes en los nódulos. Dichas fisuras son observables macroscópicamente en los nódulos experimentales y fueron observadas también en la talla experimental.

El modelado estadístico de la relación entre las variables de tamaño, desplazamiento, esquirlamiento y las melladuras; sugiere que, en el caso de estas últimas, su probabilidad de ocurrencia está vinculada al espesor del nódulo en primera instancia y posteriormente a la distancia de desplazamiento (Figura 4). Todos los nódulos con espesor mayor a 2,3 cm poseen melladuras $(n=25)$, lo mismo ocurre con aquellos que se han desplazado más de un metro $(n=2)$. Este modelo posee 

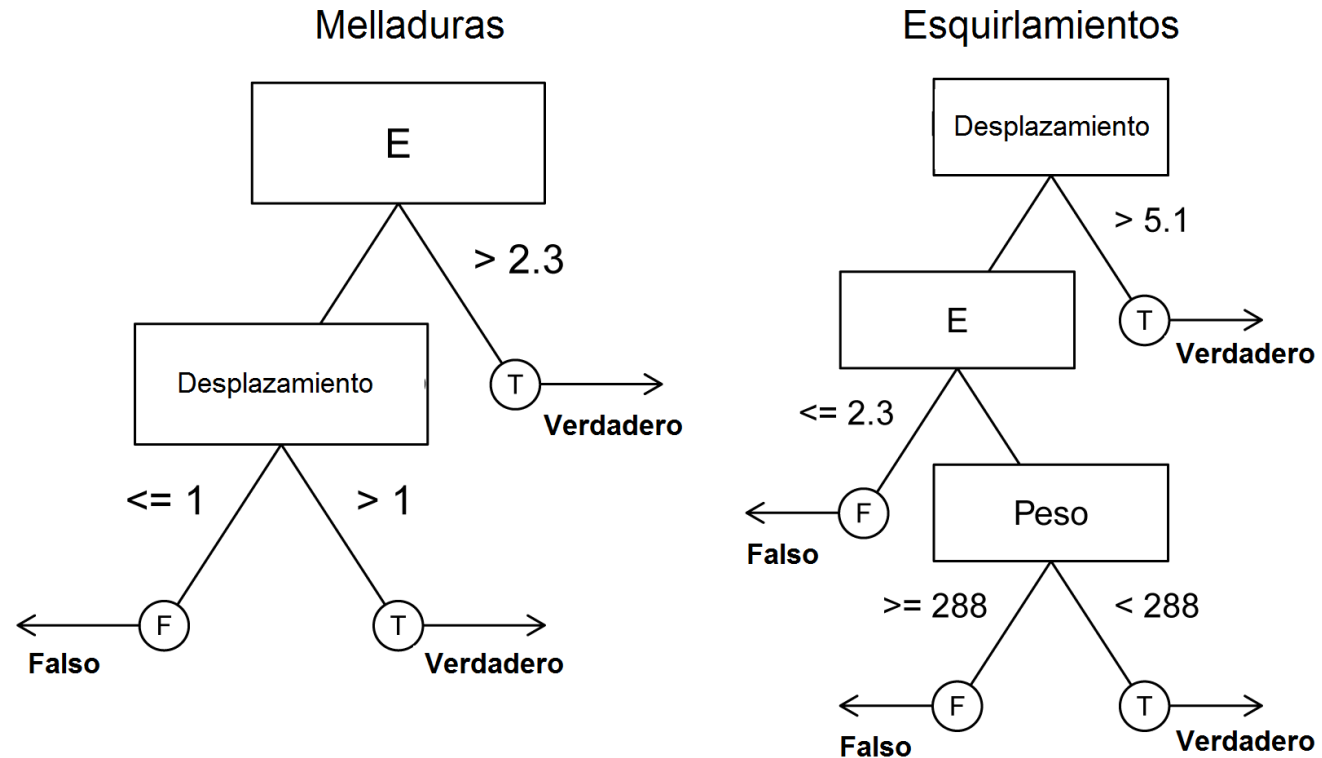

Figura 4. Árbol de decisión entre esquirlamiento, melladuras y las variables métricas seleccionadas.

Decision tree between shards, nicks dents and the selected metric variables.

un alto ajuste a los datos (sensibilidad $96 \%$, especificidad $100 \%$, precisión predictiva promedio del $98 \%$ ).

En cuanto a los esquirlamientos, el principal factor vinculado a su ocurrencia es la distancia de desplazamiento, seguido por el espesor y en menor medida, el peso (Figura 4). Aquellas rocas que se han desplazado más de $5 \mathrm{~m}$ presentan siempre melladuras $(n=9)$, por el contrario, en espesores menores a $2,3 \mathrm{~cm}$ se espera baja incidencia de este rastro (solo un caso de 5). Por último, de siete piezas mayores a $288 \mathrm{~g}$, seis poseen esquirlamientos. Este árbol posee una menor sensibilidad (83\%), una especificidad del $75 \%$ y una precisión promedio (capacidad predictiva promedio) del $79 \%$. Los resultados obtenidos en la fase experimental sugieren en general, que la ocurrencia de rastros está vinculada de manera positiva al tamaño y distancia de desplazamiento de los nódulos.

\section{Material arqueológico y experimental}

Del total del material arqueológico recuperado $(\mathrm{n}=25) 12$ artefactos proceden de superficie (48\%) y el resto de las capas de las cuales proceden los fechados radiocarbónicos antes mencionados. Dentro de esta muestra un $n=4(16 \%)$ fue determinado como ecofactos, $\mathrm{n}=20(80 \%)$ como desechos de talla (básicamente lascas y un esquirlamiento) y un solo instrumento $(0,04 \%)$, consistente en un denticulado de chert. De estos artefactos, $n=7$ (28\%) fueron clasificados inicialmente como pseudoartefactos debido a su morfología y materia prima (Figura 5).

En la muestra se identificaron siete clases de materias primas siendo la más común la calcedonia $(\mathrm{n}=9$, $36 \%$ ), y en menor medida la volcanita ácida, similar macroscópicamente a la de la pared del alero $(\mathrm{n}=7,28 \%)$ la cual está representada en su totalidad a través de los pseudoartefactos antes mencionados (Figura 5). Otras rocas en baja frecuencia son pizarra $(n=3,12 \%)$, riolita $(\mathrm{n}=2,8 \%)$ cuarzo $(\mathrm{n}=1,4 \%)$, toba silicificada $(\mathrm{n}=2,8 \%)$, sílice $(n=1,4 \%)$. Por lo que, si bien es pequeña, la muestra es llamativamente diversa.

La mayoría de estos artefactos, no poseen restos de corteza $(n=23,92 \%)$, mientras que en dos casos se conserva el $80 \%$ y el $100 \%$ respectivamente. En cuanto a los talones el más frecuente es el liso $(n=10,40 \%)$, seguido por los estallados o fragmentados $(\mathrm{n}=8,32 \%)$, corticales $(n=4,16 \%)$, filiforme $(n=2,4 \%)$ y facetado $(n=1,4 \%)$. Es interesante notar que los talones de la volcanita ácida (potencialmente pseudoartefactos) inmediatamente disponible, no presenta indicios de preparación de las plataformas, poseyendo un $42 \%$ de talones fragmentados o estallados, siendo el resto lisos y/o corticales. 


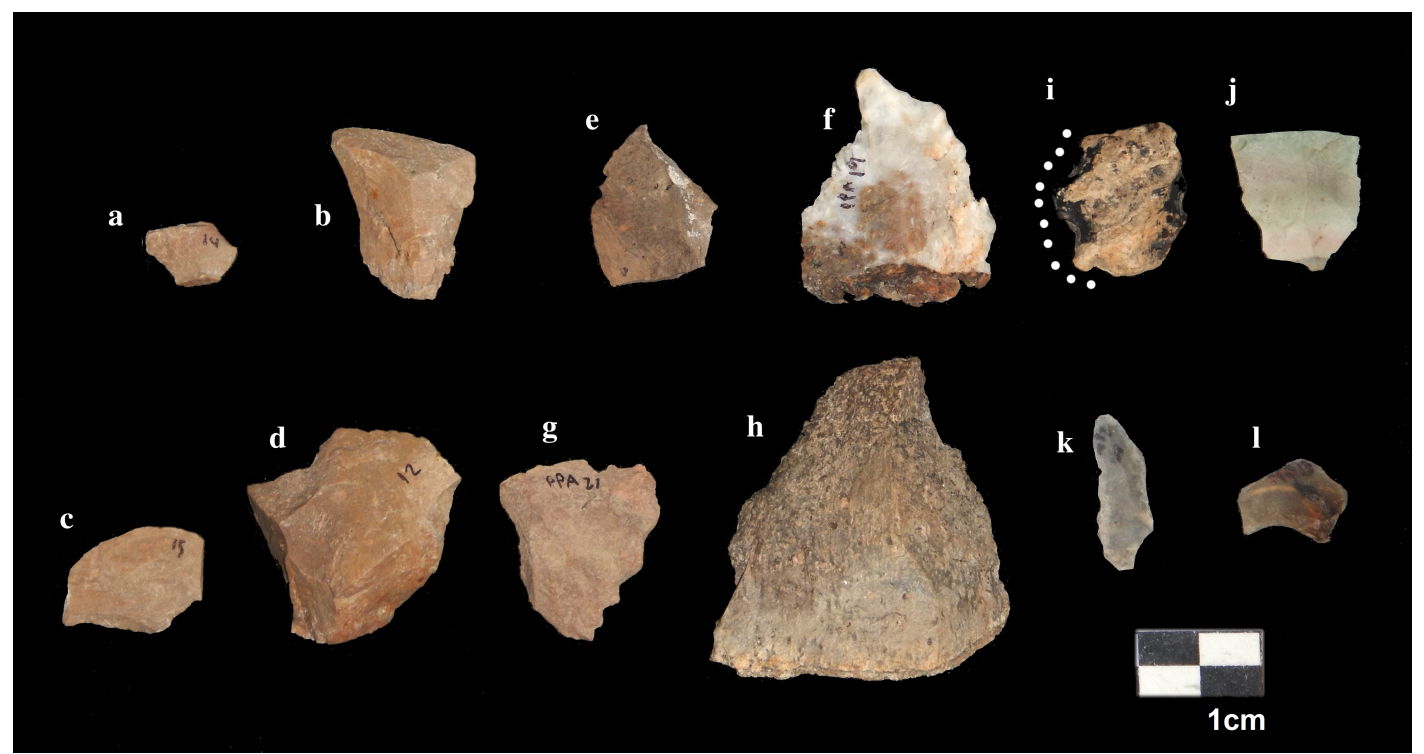

Figura 5. Lascas obtenidas de la talla experimental (a, b, c, d); pseudoartefactos (e, f, g, h); material arqueológico: denticulado (i), la línea de puntos indica el filo; lascas ( $\mathrm{j}, \mathrm{k}, \mathrm{l})$.

Flakes obtained from the experimental knapping ( $a, b, c, d)$; pseudo-artifacts $(e, f, g, h)$; archaeological material: denticulate (i), the dotted line indicates the cutting edge; flakes $(j, k, l)$.

La calidad de las rocas es en general buena o muy buena $(n=19,76 \%)$ el $4 \%$ restante está compuesto por vulcanitas ácidas de calidad regular pertenecientes a la misma formación del alero (aunque no necesariamente provenientes de la pared misma). El tamaño de los artefactos enteros (Tabla 2) sugiere variabilidad en estos valores, siendo los fragmentos más pequeños de 0,1 g y los más pesados de 42 g (media 4,7 g, desvío estándar $11,97 \mathrm{~g}$ ). Esto se relaciona tanto a la presencia de microlascas (de calcedonia de calidad alta) como lascas grandes o pseudoartefactos de vulcanita ácida.

\section{Material experimental}

Tal como mencionamos anteriormente, el material experimental proviene de la pared del alero (de calidad buena a regular) y de la parte superior del mismo (de

Tabla 2. Correlación lineal entre las variables métricas de los nódulos analizados y el desplazamiento observado (triángulo inferior). Triangulo inferior, probabilidades obtenidas. *correlación significativa.

Linear correlation between the metric variables of the analyzed nodules and the observed displacement (lower triangle). Lower triangle, obtained probabilities. *significant correlation.

\begin{tabular}{lccccc}
\hline \multicolumn{1}{c}{ Variables } & Largo & Ancho & Espesor & Peso & Desplazamiento \\
\hline Largo & & 0,02 & 0,04 & 0,13 & 0,64 \\
Ancho & 0,42 & & 0 & 0,12 & 0,38 \\
Espesor & 0,38 & 0,63 & & 0,01 & $0,01^{*}$ \\
Peso & 0,28 & 0,29 & 0,48 & & 0,1 \\
Desplazamiento & $-0,09$ & 0,17 & 0,47 & 0,3 & \\
\hline
\end{tabular}

calidad mala). Solo en el nódulo tallado proveniente de la pared del alero de mayor calidad se produjeron subproductos de talla con atributos claramente reconocibles. De éste provienen 15 lascas producidas en la talla, de las cuales $n=6(40 \%)$ se fragmentaron durante la extracción, de éstas el $66 \%$ presenta talones lisos $(n=10)$, seguido por puntiformes $(n=3,20 \%)$ $\mathrm{y}$ estallados $(\mathrm{n}=2,13 \%)$. Es interesante notar que esta proporción de talones estallados es menor a la observada arqueológicamente, aunque en ambos casos, predominan los lisos.

En cuanto al tamaño la muestra experimental presenta una menor dispersión de las variables métricas (Tabla 3). Si bien ambas muestras son reducidas, es posible compararlas de forma preliminar a partir de sus coeficientes de variación (desviación estándar/media).

Tal como se observa en la Tabla 3, el coeficiente de variación de las variables métricas para la muestra arqueológica se encuentra entre un 50\% y un $100 \%$ (o mayor aún) en relación con la experimental. Esta diferencia es especialmente notable en la superficie, volumen y peso de los desechos arqueológicos enteros.

Creemos que al comparar ambas muestras es posible extraer algunas tendencias generales. La muestra arqueológica posee una notable variación métrica y morfológica, aunque está dominada por pequeños desechos de talla que sugieren estados avanzados de reducción, así como tareas de mantenimiento o retoque, donde el descarte de instrumentos ha sido inusual. $\mathrm{La}$ composición de las rocas en esta muestra sugiere el empleo preferencial de rocas de buena y muy buena 
Tabla 3. Variables métricas de los artefactos líticos. Coef. de var.: coeficiente de variación.

Metric variables of lithic artifacts. Coef. of var: coefficient of variation.

\begin{tabular}{lllllll}
\hline & $\begin{array}{c}\text { Largo } \\
(\mathrm{mm})\end{array}$ & $\begin{array}{c}\text { Ancho } \\
(\mathrm{mm})\end{array}$ & $\begin{array}{c}\text { Espesor } \\
(\mathrm{mm})\end{array}$ & $\begin{array}{c}\text { Superficie } \\
(\mathrm{mm})\end{array}$ & $\begin{array}{c}\text { Volumen } \\
\left(\mathrm{mm}^{3}\right)\end{array}$ & $\begin{array}{c}\text { Peso } \\
(\mathrm{g})\end{array}$ \\
\hline Experimental & & & & & & \\
\hline Mínimo & 10 & 10 & 2 & 150 & 0,15 & 0,50 \\
Máximo & 30 & 30 & 10 & 810 & 0,82 & 6,90 \\
Media & 21,33 & 18,22 & 5,56 & 413,22 & 0,42 & 2,48 \\
Desvío estándar & 7,26 & 6,78 & 2,46 & 257,96 & 0,26 & 2,11 \\
Coef de var & 34,04 & 37,20 & 44,19 & 62,43 & 61,91 & 85,23 \\
\hline \multicolumn{1}{c}{ Arqueológico } & & & & & & \\
\hline Mínimo & 8 & 6 & 1 & 48 & 0,05 & 0,10 \\
Máximo & 53 & 50 & 20 & 2650 & 53 & 42,10 \\
Media & 21,75 & 16,58 & 5 & 509 & 6,16 & 4,68 \\
Desvío estándar & 13,40 & 13,15 & 5,29 & 763,44 & 15,18 & 11,97 \\
Coef. de var. & 61,63 & 79,27 & 105,83 & 149,99 & 246,25 & 256,08 \\
\hline
\end{tabular}

calidad, aunque la presencia de potenciales desechos de volcanita ácida indicaría el uso de materia prima inmediatamente disponible de calidad regular.

La muestra experimental, por otro lado, indica que las volcanitas de mejor calidad son regulares para la talla, ya que los nódulos presentan sectores con distinta cristalización y múltiples planos de debilidad que tornan la talla altamente impredecible. Sin embargo, es posible extraer subproductos con atributos de talla, morfológicamente similares a los observados arqueológicamente. Lo que plantea un posible problema de equifinalidad para este tipo de materiales, si bien no hemos observado que se generen durante los experimentos de caída, lo que discutiremos más ampliamente a continuación.

\section{Discusión}

Este trabajo nos permitió evaluar la dinámica del alero y su talud, así como los procesos que actúan en la acumulación, dispersión y fractura de material en este espacio del sector costero oeste del golfo San Matías.

En primer lugar, el material experimental sugiere que la materia prima que constituye la Punta Pórfido y en particular el sector del alero es de calidad predominantemente baja con afloramientos espacialmente acotados de mejor calidad. Por otro lado, el desarrollo de la talla controlada sobre nódulos de menor calidad nos permite afirmar que ésta no presenta fractura concoidea ni genera subproductos con atributos tecnológicos, sino que se fragmenta de manera irregular a lo largo de los planos de debilidad preexistentes en la roca. La vulcanita de mayor calidad en cambio presenta fractura concoidea, aunque los núcleos tienden a fragmentarse de forma impredecible durante la talla, siguiendo fisuras, planos de debilidad y nódulos cristalinos de la roca. En este caso es posible identificar plataformas de percusión, estrías y charnelas sobre fragmentos de núcleos, asimismo, una baja proporción de los desechos generados son lascas con filos naturales potencialmente utilizables.

La experiencia de caída de rocas, por otro lado, permitió tener una aproximación a la manera en que este fenómeno puede haber aportado a la formación del depósito. Bloques más grandes, de forma relativamente redondeada pueden haberse desplazado a mayor distancia, depositándose al borde del talud, sin generar pseudoartefactos. Sin embargo, es esperable que, en estos casos, se produzcan astillamientos y melladuras en los bloques cuanto mayor sea la distancia que desplacen a lo largo de la pendiente. Es posible que el modelo generado experimentalmente explique, al menos en parte, la baja frecuencia de bloques recuperados durante la excavación, ya que estos se habrían depositado preferencialmente al pie del talud. Por consiguiente, los resultados obtenidos apuntan a que la pendiente natural del alero ha facilitado la reptación de los materiales por gravedad a lo largo del tiempo (Rick 1976). Esto último podría contrastarse realizando sondeos al pie del talud, donde deberían poder recuperarse artefactos, nódulos y pseudoartefactos de mayor tamaño.

En segundo lugar, la evidencia recuperada en el alero, indica una muy baja tasa de depositación antrópica, por lo que el depósito habría sido generado mayormente por procesos endógenos, como la meteorización física y química de la pared y techo del alero. Las características del registro sugieren un uso esporádico y poco intenso de este espacio a lo largo de unos 6000 años AP. La existencia de lascas y microlascas y la ausencia de núcleos o productos corticales, podría 
relacionarse con un uso puntual del alero, donde se reactivaron instrumentos o se realizaron las fases finales del proceso de reducción quizás en una forma oportunística y en donde puede haberse empleado parte de la roca aflorante del alero de forma inmediata. En este contexto, hay un predominio de desechos y materia prima que no proviene directamente del alero, sino de fuentes secundarias de aprovisionamiento locales. A unos escasos $100 \mathrm{~m}$ se detectaron acumulaciones de material superficial, principalmente desechos de talla, que están siendo analizados. En esta muestra se detectaron un núcleo de vulcanita ácida de calidad buena, macroscópicamente similar a la de la pared del alero, así como lascas de cuarzo también disponible de forma local en venas aflorantes de la misma formación (Alberti y Cardillo 2015). Los materiales analizados provenientes de muestreos superficiales y del locus Punta Pórfido sondeo 1 ubicado a unos 650 metros hacia el SO, muestran una amplia diversidad de materiales líticos (Borella et al. 2015) dentro de los que se destacan las pesas de línea o red, así como puntas de proyectil de variada morfología en sílice y obsidiana. En conjunto, las evidencias recuperadas al momento en esta localidad señalan redundancia ocupacional, la explotación de diversos recursos faunísticos y gran diversidad artefactual; así como de las materias primas explotadas, lo que contrasta con la evidencia recuperada hasta el momento en este locus (Alberti y Cardillo 2015; Borella et al. 2015).

En cuanto a los potenciales artefactos o pseudoartefactos, no fue posible identificar rasgos o atributos que confirmaran la presencia de estos en el sitio. Lo que sí nos permitió la experimentación y el empleo de árboles de decisión fue acotar bajo qué condiciones esperamos detectar los distintos indicadores estudiados (esquirlamientos, melladuras y desplazamiento diferencial), así como generar expectativas muy concretas sobre el comportamiento de los bloques en relación al talud del alero. Estas modificaciones postdepositacionales observadas mediante el trabajo actualístico, nos brindaron la posibilidad de entender la forma en que estos bloques se depositan en el espacio del locus, tanto en la boca del alero como en el talud. Los resultados obtenidos hasta el momento apuntan a que resulta poco plausible que la caída de rocas sea un agente que forme lascas análogas a las producidas por la talla controlada. Más bien, los efectos morfológicos referidos a la caída de rocas se encontraron asociados principalmente a la producción de esquirlas y melladuras. Siguiendo a Borrazzo (2016) creemos que este caso de estudio nos permitió realizar una serie de afirmaciones del orden de lo tafonómico teniendo en cuenta el escenario planteado hasta el momento, en donde la materia prima de los potenciales artefactos se encuentra naturalmente disponible en el contexto del alero. En este caso, sería esperable recuperar núcleos o fragmentos de núcleos con indicios de plataformas de percusión tal como se observó en la talla experimental. Asimismo, si bien logramos avanzar en el estado de conocimiento, resta trabajo para realizar a futuro. Por ejemplo, sistematizar estudios en el talud y profundizar en el estudio e identificación de los atributos de pseudoartefactos. Creemos que el caso bajo estudio nos representa un desafío para seguir evaluando esta línea de investigación actualística en vista a poder ajustar nuestros resultados e interpretaciones sobre las ocupaciones humanas en el pasado.

\section{Conclusiones}

El objetivo central de este trabajo consistió en identificar los efectos morfológicos de los procesos físicos, como así también la identificación de atributos y rasgos que nos permitan reconocer la variabilidad en el registro lítico en un contexto de alero, tomando como caso particular el Alero 2 de Punta Pórfido. La integración de las líneas de evidencia arqueológica y experimental señalan que la morfología del alero y los procesos endógenos de meteorización y caída de bloques han sido los principales agentes de formación, en un espacio que fue utilizado de forma puntual y esporádica a lo largo del tiempo. Es posible entonces, afirmar en esta primera instancia, que al menos una fracción de los potenciales pseudoartefactos recuperados puede haber sido generada de manera oportunística con material endógeno del alero.

Por otro lado, creemos que el principal aporte de este trabajo es de índole metodológica en lo referido a los estudios de tafonomía lítica y a los análisis experimentales en general. En este sentido, los resultados obtenidos señalan la utilidad de los árboles de decisión para evaluar el rol de los distintos agentes tafonómicos, así como para generar expectativas de naturaleza cuantitativa contrastables, tal como fue señalado por Weitzel et al. (2014), empleando métodos similares.

Por último, remarcamos la utilidad de las observaciones locales de los contextos arqueológicos y no arqueológicos, en conjunto con la experimentación, como herramientas para construir marcos de referencia. Esperamos que el avance de las excavaciones en este sector permita identificar subsectores con mayor densidad artefactual y profundizar en el conocimiento en que este espacio de características únicas para la costa rionegrina.

Agradecimientos: Este trabajo ha sido realizado como parte de la beca doctoral CONICET de la autora y dentro de los proyectos PIP. CONICET 2012-2014 dirigido por la Dra. Florencia Borella y PICT-2013-1128 dirigido por el Dr. Cristián Favier Dubois. Agradecemos especialmente a la Dra. 
Florencia Borella por sus comentarios y sugerencias, al Dr. Cristian Favier Dubois y Florencia Borella por su apoyo durante la realización de las tareas de campo y a la Dra. Karen Borrazzo por el apoyo y discusiones que aportaron al desarrollo del trabajo.
A Cultura Río Negro y Áreas Naturales Protegidas de Río Negro por los permisos de trabajo otorgados. Agradecemos especialmente al comité editorial y a los evaluadores cuyos comentarios y sugerencias mejoraron sustancialmente este trabajo.

\section{Referencias Citadas}

Alberti, J. y M. Cardillo 2015. Primary and secondary lithic raw material sources along the western coast of San Matías Gulf (Río Negro province, Argentina): A first approach to their spatial variability. Quaternary Internacional 373:63-71.

Alberti, J., M. Cardillo y C. Favier Dubois 2015. Fuentes de materias primas líticas en la costa del golfo San Matías (provincia de Río Negro, Argentina). Una síntesis regional. Intersecciones en Antropología Volumen Especial 2:27-37.

Alberti, J. y V. Fernández 2015. Propuesta clasificatoria para las materias primas líticas en Patagonia (Argentina). Arqueología 21:211-233.

Aschero, C. 1983. Ensayo para una clasificación morfológica de artefactos líticos. Apéndices A y B. Cátedra de Ergología y Tecnología. Facultad de Filosofía y Letras, Universidad de Buenos Aires, Buenos Aires.

Borella, F. y M. Cardillo (compil.) 2011. Arqueología de Pescadores y Marisqueadores en Nordpatagonia: Descifrando un Registro de más de 6000 años. Editorial Dunken, Buenos Aires.

Borella, F., M. Cardillo, C. Favier Dubois y J. Alberti 2015. Nuevas investigaciones arqueológicas entre Punta Pórfido y Punta Odriozola: implicancias para el entendimiento de la dinámica de las ocupaciones humanas en la costa oeste del golfo San Matías (Río Negro). Relaciones de la Sociedad Argentina de Antropología XL (1):233-252.

Borrazzo, K. 2011. Tafonomía lítica y pseudoartefactos: el caso de la península El Páramo (Tierra del Fuego, Argentina) Intersecciones en Antropología 12:155-167.

Borrazzo, K. 2016. Lithic taphonomy in desert environments: contributions from Fuego-Patagonia (Southern South America). Quaternary International 422:19-28.

Borrero, L.A. 2015. Con lo mínimo: los debates sobre el poblamiento de América del Sur. Intersecciones en Antropología 16 (1):5-38.

Cardillo, M. y F. Scartascini 2011. Diversidad artefactual y explotación de materias primas en la costa norpatagónica, golfo San Matías, Río Negro. En Arqueología de Pescadores y Marisqueadores en Nordpatagonia. Descifrando un Registro de más de 6000 años, compilado por F. Borella y M. Cardillo, pp. 68-86. Editorial Dunken, Buenos Aires.

De Alba, E. 1964. Descripción Geológica de la Hoja 41jSierra Grande. Ministerio de Economía de la Nación, Secretaría de Industria y Minería

Favier Dubois, C.M. 1997. Indicadores de tasas de depositación relativas en el registro arqueológico, su aplicación en sitios de Fuego Patagonia. Arqueología 7:51-75.
Favier Doubois, C. y F. Borella 2011. Contrastes en la costa del golfo: una aproximación al estudio del uso humano del litoral rionegrino. En Arqueología de Pescadores y Marisqueadores en Nordpatagonia. Descifrando un Registro de más de 6.000 Años, editado por F. Borella y M. Cardillo, pp. 13-42. Editorial Dunken, Buenos Aires.

Favier Dubois, C.M.,F. Borella y M. Cardillo 2017. Cronologías de ocupación en la costa oeste del golfo San Matías (Río Negro): factores geomorfológicos involucrados. X Jornadas de Arqueología de la Patagonia: Libro de Resúmenes, pp. 34. Instituto de Diversidad y Evolución Austral, Puerto Madryn.

Favier Dubois, C., F. Borella, L. Manzi, M. Cardillo, S. Lanzellotti, F. Scartascini, C. Mariano y E. Borges Vaz 2008. Aproximación regional al registro arqueológico de la costa rionegrina. En Arqueología de la Costa Patagónica. Perspectivas para la Conservación, editado por I. Cruz y S. Caracotche, pp. 50-68. Universidad Nacional de la Patagonia Austral, Río Gallegos

Favier Dubois, C.M. y A.J.T. Jull 2018. Marine reservoir effect values from shell-middens of San Matías Gulf, northern Patagonia (Argentina): a 5000-yr record. Radiocarbon 60 (2):419-424.

Garvey, R. y F. Mena 2016. Confronting complexities of artifactgeofact debates: re-analysis of a coarse volcanic rock assemblage from Chilean Patagonia. Lithic Technology 41 (2):114-129.

Gigerenzer, G., P.M. Todd y T. ABC Research Group 1999. Simple Heuristics that Make Us Smart. Oxford University Press, Oxford

González Díaz, E. y E. Malagnino 1984. Geomorfología de la provincia de Río Negro. Actas del IX Congreso Geológico Argentino, p. 159. San Carlos de Bariloche.

Haynes, V. 1973. The Calico Site: Artifacts or geofacts? Science 181 (4097):305-310.

Hogg, A.G., P.G. Blackwell, M. Niu, C.E. Buck, T.P. Guilderson, T.J. Heaton, J.G. Palmer, P.J. Reimer, R.W. Reimer, C.S.M Turney y S.R.H. Zimmerman 2013. SHCal13 Southern Hemisphere calibration, 0-50,000 years cal BP. Radiocarbon 55 (4):1889-1903.

Jackson, D. y C. García 2005. Los instrumentos líticos de las ocupaciones tempranas de Marifilo 1. Boletín de la Sociedad Chilena de Arqueología 38:71-78.

Lyman, R.L. 2004. The concept of equifinality in taphonomy. Journal of Taphonomy 2 (1):15-26.

Lin, S.C., Z. Rezek y H.L. Dibble 2017. Experimental design and experimental inference in stone artifact archaeology. Journal of Archaeological Method and Theory 25 (3):663-688. 
Lubinski, P.M., K. Terry y P.T. McCutcheon 2014. Comparative methods for distinguishing flakes from geofacts: a case study from the Wenas Creek Mammoth site. Journal of Archaeological Science 52:308-320.

Manzi, L., C. Favier Dubois y F. Borella 2009. Identificación de agentes perturbadores y estrategias tendientes a la conservación del patrimonio arqueológico en la costa del Golfo de San Matías, provincia de Río Negro. Intersecciones en Antropología 10:3-16.

Nash, D. T. 1993. Distinguishing stone artifacts from naturefacts created by rockfall processes. En Formation Processes in Archaeological Context, editado por P. Goldberg, D.T. Nash y M.D. Petraglia, pp. 125-138. Madison: Monographs in World Prehistory $\mathrm{N}^{\mathrm{o}} 17$, Prehistory Press.

Phillips, N.D., N. Hansjörg, W. Jan y G. Wolfgang 2017. FFTrees: A toolbox to create, visualize, and evaluate Fast-and-Frugal Decision Trees. Judgment and Decision Making 12 (4):344-368.

Prentiss, A.M., M.J. Walsh, K.D. Barnett, M.M. Murphy y J. Kuenstle 2015. The coarse volcanic rock industry at Rio Ibáñez
6 west, Aisén Region, Patagonian Chile. Lithic Technology 40 (2):112-127.

Rick, J.W. 1976. Downslope movement and archaeological intrasite spatial analysis. American Antiquity 41 (2):133-144.

Song, Y.Y. y L.U Ying 2015. Decision tree methods: applications for classification and prediction. Shanghai Archives of Psychiatry 27(2):130.

Waters, M.R. 1992. Principles of Geoarchaeology: A North American Perspective. University of Arizona Press, Tucson.

Weitzel, C., K. Borrazzo, A. Ceraso y C. Balirán 2014. Trampling fragmentation potential of lithic artifacts: an experimental approach. Intersecciones en Antropología. Volumen Especial 1:97-110.

Wood, W.R. y D.L. Johnson 1982. A survey of disturbance processes in archaeological site formation. En Advances in Archaeological Method and Theory: Selections for Students 1-4:539-605

\section{Nota}

1 "A particular pattern in material remains can be created by entirely different sets of causal processes" (Cunningham 2003:392 en Lyman 2004:25). 
